tung von Common PKI: „Für den Bereich der Heilberufsausweise ist Common PKI fest in die HPC-Spezifikation verankert. Die HPC-Spezifikation definiert die Chipkarten-Plattform und die Zertifikatsprofile aller Heilberufler im deutschen Gesundheitswesen. Für den Bereich der elektronischen Arztausweise stellt die Common PKI Spezifikation das Maß aller Dinge dar."

Weiterhin besteht die Möglichkeit, das Protokoll von einem anerkannten Prüflabor vorab prüfen zu lassen. Dies wird auf der Webseite www.common-pki.org deutlich kenntlich gemacht. Bisherige Common PKI Siegel werden in dieser Weise weiter auf der Webseite geführt. Im Ergebnis können Hersteller jetzt wählen, ob sie die Tests für eine Produktregistrierung selber durchführen oder von einem Prüflabor bestätigen lassen wollen. Dieses Verfahren ersetzt das bisherige Common PKI Siegel, bei dem die Prüfung durch ein Prüflabor in jedem Fall beinhaltet war.

Eine Erläuterung des Verfahrens findet sich auf www.commonpki.org. Auch das Common PKI Testbed kann dort bestellt werden.

\section{TÜV Informationstechnik als IT-Sicherheitsdienstleister zertifiziert}

Das Bundesamt für Sicherheit in der Informationstechnik (BSI) hat die TÜV Informationstechnik GmbH (TÜViT) als IT-Sicherheitsdienstleister im Anwendungsbereich Informationssicherheitsrevision (IS-Revision) und Informationssicherheitsberatung (IS-Beratung) zertifiziert. Das Zertifikat zeichnet TÜViT als vertrauenswürdigen und kompetenten IT-Sicherheitsdienstleister aus und befähigt das Unternehmen insbesondere, qualifizierte Informationssicherheitsrevisionen im Rahmen des Umsetzungsplanes (UP) Bund durchzuführen.

IS-Revision ist ein wichtiger Bestandteil eines erfolgreichen Informationssicherheitsmanagements. Nur durch die regelmäßige Überprüfung der etablierten Sicherheitsmaßnahmen und -prozesse können Aussagen über deren Wirksamkeit, Aktualität, Vollständigkeit und Angemessenheit und damit über den aktuellen Zustand der Informationssicherheit getroffen werden. Die IS-Revision ist somit ein Werkzeug, mit dem ein angemessenes Sicherheitsniveau in einer Institution erreicht und aufrechterhalten werden kann.

\section{Beirat der Allianz für Cyber-Sicherheit hat sich konstituiert}

Im Rahmen des 13. Deutschen IT-Sicherheitskongresses, den das Bundesamt für Sicherheit in der Informationstechnik (BSI) Bonn ausrichtet, hat am 14.05.2013 die konstituierende Sitzung des Beirats der Allianz für Cyber-Sicherheit stattgefunden. Aufgabe des Beirats ist es, die geleistete Arbeit der Allianz für Cyber-Sicherheit kritisch zu reflektieren und Impulse für die zukünftigen Themen und Schwerpunkte zu geben. Der Beirat nimmt dabei keine operative, sondern eine beratende Funktion wahr. Mitglieder des Beirats sind:

- Prof. Dieter Kempf, Präsident des Bundesverbands Informationswirtschaft, Telekommunikation und neue Medien (BITKOM)

- Elmar Rothenwöhrer, Vertreter des Bundesverbandes der Deutschen Industrie (BDI) im Beirat der Allianz für Cybersicherheit
- Dr. Klaus Mittelbach, Vorsitzender der Geschäftsführung des Zentralverbands Elektrotechnik- und Elektronikindustrie (ZVEI)

- Dr. Thomas Endres, Vorsitzender des Präsidiums des VOICE - Verband der IT-Anwender

- Martin Schallbruch, IT-Direktor im Bundesministerium des Innern (BMI)

- Michael Hange, Präsident des Bundesamts für Sicherheit in der Informationstechnik (BSI)

In Zukunft sollen auch Vertreter der Partner, Teilnehmer und Multiplikatoren der Allianz für Cyber-Sicherheit sowie weitere Verbände dem Beirat angehören. Zum Vorsitzenden des Beirats wurde Prof. Dieter Kempf gewählt.

\section{Bundesrat stimmt dem E-Government-Gesetz zu}

Trotz zahlreicher kritischer Stellungnahmen - insbesondere aus dem Kreis der Datenschutzbeauftragten - hat der Bundesrat am 07.06.2013 dem zuvor bereits vom Bundestag beschlossenen E-Government-Gesetz zugestimmt.

Ziel des E-Government-Gesetzes ist es, durch den Abbau bundesrechtlicher Hindernisse die elektronische Kommunikation mit der Verwaltung zu erleichtern. Das Gesetz soll es dadurch Bund, Ländern und Kommunen ermöglichen, einfachere, nutzerfreundlichere und effizientere elektronische Verwaltungsdienste anzubieten. Elektronische Verwaltungsdienste ermöglichen es Bürgern und Unternehmen, unabhängig von Öffnungs-und Sprechzeiten und ortsunabhängig mit Behörden zu kommunizieren. Der Gang zum Amt wird in vielen Fällen überflüssig.

Das E-Government-Gesetz wird vorbehaltlich einiger Ausnahmen am Tag nach der Verkündung des Gesetzes im Bundesgesetzblatt in Kraft treten. Die Ausnahmen treten wie folgt zeitlich gestaffelt in Kraft:

\section{Juli 2014:}

- Pflicht der Behörden von Bund und Ländern, elektronische Dokumente anzunehmen, auch dann, wenn diese mit einer qualifizierten elektronischen Signatur versehen sind („Pflicht zur Zugangseröffnung").

- in der Verwaltung des Bundes die Möglichkeit, De-Mail zum Ersatz der Schriftform einzusetzen. Hinweis: Die Möglichkeit, Online-Formulare in Verbindung mit dem elektronischen Identitätsnachweis des neuen Personalausweises zum Ersatz der Schriftform zu nutzen, besteht in der Verwaltung des Bundes bereits am Tag nach der Verkündung des Gesetzes.

\section{Januar 2015:}

- Pflicht der Bundesbehörden, die Nutzung des elektronischen Identitätsnachweises nach dem Personalausweisgesetz zu ermöglichen und dafür die auf Seiten der Behörden notwendige Infrastruktur bereitzustellen (z. B. Erwerb von sogenannten Berechtigungszertifikaten nach dem Personalausweisgesetz).

- die sich aus Artikel 1 § 14 des Gesetzes ergebenden Pflichten der Behörden von Bund und Ländern zur Georeferenzierung.

\section{ein Kalenderjahr}

nach der Aufnahme des Betriebs des zentral für die Bundesverwaltung angebotenen IT-Verfahrens, über das De-Mail-Dienste für Bundesbehörden angeboten werden:

- Pflicht für Bundesbehörden, per De-Mail erreichbar zu sein. 